\title{
Lagrangian coherent structures and mixing in two-dimensional turbulence
}

\author{
G. Haller*, G. Yuan \\ Division of Applied Mathematics, Lefschetz Center for Dynamical Systems, Brown University, Providence, RI 02912, USA \\ Received 11 February 2000; received in revised form 6 June 2000; accepted 10 July 2000 \\ Communicated by U. Frisch
}

\begin{abstract}
We introduce a Lagrangian definition for the boundaries of coherent structures in two-dimensional turbulence. The boundaries are defined as material lines that are linearly stable or unstable for longer times than any of their neighbors. Such material lines are responsible for stretching and folding in the mixing of passive tracers. We derive an analytic criterion that can be used to extract coherent structures with high precision from numerical or experimental data sets. The criterion provides a rigorous link between the Lagrangian concept of hyperbolicity, the Okubo-Weiss criterion, and vortex boundaries. We apply the results to simulations of two-dimensional barotropic turbulence. (c) 2000 Elsevier Science B.V. All rights reserved.
\end{abstract}

Keywords: Coherent structures; Mixing two-dimensional turbulence; Okubo-Weiss criterion; Invariant manifolds

\section{Introduction}

It is well known that in two-dimensional turbulent fluid flows coherent structures tend to emerge. While the existence of such structures is clear from visual observations of turbulence, their physical description is not at all obvious. For instance, a coherent structure can be characterized as a region of concentrated vorticity that retains its identity for long times (see, e.g., [42]). Alternatively, coherent structures can be viewed as energetically dominant recurrent patterns (see, e.g., [20]). Finally, coherent structures can also be thought of as sets of fluid particles with distinct statistical properties (see, e.g., [14]). While emphasizing different physical aspects of the spatial inhomogeneity of turbulence, all these approaches fall into one of the two basic categories: Eulerian or Lagrangian.

\footnotetext{
* Corresponding author.

E-mail address: haller@cfm.brown.edu (G. Haller).
}

The Eulerian approach to coherent structures aims at partitioning the flow based on the instantaneous distribution of a scalar field, such as the vorticity, kinetic energy, enstrophy, or the strain. For instance, coherent vortices can be identified as regions with vorticity over a small threshold (see [3,7]), regions bounded by curves of maximal (potential) vorticity gradient (see $[22,26]$ ), or regions where vorticity dominates strain (see, e.g., [7,9,27,32,48]). A refinement of the vorticity-based definition, requiring near-axisymmetry for coherent vortices, is given in [28], while refinements and improvements of the strain-vorticity ratio based criterion (the Okubo-Weiss criterion) are given in $[6,14,21]$. While all these Eulerian criteria tend to yield qualitatively similar results, the individual structures they identify typically differ. Nevertheless, formulated in terms of level sets and gradients of scalar fields, each such criterion is quantitative and hence can be implemented systematically. 
The Lagrangian approach to the coherent structures of two-dimensional turbulence is concerned with patterns emerging from the advection of passive tracers. In particular, coherent vortices have been studied in terms of absolute and relative single particle dispersion (see, e.g., $[4,42])$ and passive tracer dynamics has been statistically related to the presence of Eulerian coherent structures obtained from the Okubo-Weiss criterion (see [14]). In addition, particle dynamics in point vortex models have been compared to those induced by coherent vortices in turbulence (see, e.g., $[1,2,5,42]$ or [42] for a review). Finally, finite-time Lagrangian Lyapunov exponents have been employed to locate dynamically distinguished regions in geophysical flow data (see [37,38]). In most of these studies coherent structures have been fairly vaguely defined, and hence their systematic detection has not been pursued.

Lagrangian approaches to turbulence are strongly influenced by the observation that the dynamical behavior of tracers is more dramatically affected by phase space geometry (such as the distance of tracers from vortex cores) than by the detailed time history of the velocity field (see, e.g., [14]). This gives some hope that geometric structures familiar from simple models of chaotic advection might have some relevance in the description of the coherent building blocks of two-dimensional turbulence. In time-periodic or quasiperiodic, analytically defined advection models it is enough to know the velocity field for finite times to reproduce its recurrent infinite-time history. As a result, the boundaries of dynamic coherent structures can be located through stable and unstable manifolds of appropriate Poincaré maps, and the transport associated with these structures can be quantified via lobes (see, e.g., [10,25,34,44-47,50,51] for surveys). This quantitative power of the Lagrangian approach, however, is lost for turbulent flows with complicated spatial and temporal structure. For such flows, one would need to know the velocity field for infinite times to define stable and unstable manifolds and lobes. For this reason, the definition, location, and extraction of coherent structures has not been clarified in geometric terms, and the description of tracer dynamics has remained statistical, based on a primarily Eulerian view on coherent structures [14]. Yet there is a strong need for advances in this area. Beyond the unresolved theoretical issues, much of the available observational data on geophysical turbulence is provided by floats, which requires the use of Lagrangian tools in the identification of structures of interest (see, e.g., [35]). In addition, studying the details of turbulent tracer mixing is of great importance in atmospheric applications (see, e.g., [36]), industrial flow control problems (see, e.g., [31]), and many other areas.

In this paper we propose a Lagrangian description of coherent structures as regions of qualitatively different tracer dynamics. Such regions can be best approached by following the mixing of passive tracers. An initially regular passive tracer blob tends to reveal nearby coherent structures through stretching, thinning, and folding around them. As we argue below, these dynamical events are due to the presence of linearly stable and unstable material lines in the flow. For lack of regular time dependence, in a turbulent flow one would need to track material lines for all times in order to verify their stability or instability in the conventional sense. Since this is not feasible, we introduce a concept of finite-time stability and instability for material lines. This definition is motivated by the concept of uniform normal hyperbolicity from dynamical systems and is phrased for surfaces spanned by evolving material lines in space-time. We then define Lagrangian coherent structure boundaries as material lines with locally the longest or shortest stability or instability time. We show a direct connection between the dynamical boundaries we propose and those one can (approximately) infer from single particle dispersion. In addition, the stable (unstable) material lines coincide with unstable (stable) manifolds of Poincaré maps in flows with periodic time dependence, thus providing a natural link between geometric ideas from chaotic advection and turbulence.

To locate coherent structure boundaries in given data sets, we derive an analytic result. According to Theorem 1, a material line is a coherent structure boundary if it satisfies the hyperbolicity condition of the Okubo-Weiss criterion along with another condition, for locally the longest time in the flow. We use this result to devise a numerical algorithm that ex- 
tracts coherent structure boundaries from experimental or numerical data sets. To test the algorithm, we conduct a Lagrangian study of a velocity field arising from simulations of quasigeostrophic turbulence. In this velocity field, we isolate Lagrangian coherent structure boundaries using our analytic criterion, and show that they coincide with the boundaries suggested by relative and absolute dispersion statistics. However, our dynamical boundaries are exactly defined and sharp, and are also available in regions, where the statistically predicted boundaries are missing or appear too scattered. We also show how coherent structures of different strength can be located, which gives a tool to explore the internal structure of background turbulence.

\section{Motivation: turbulent mixing of passive tracers}

Our main interest will be the partitioning of a two-dimensional fluid flow into regions that display different dynamical behavior. Such dynamical regions or "Lagrangian" coherent structures can be, e.g., regions of sustained stirring with a given orientation, or sustained translational motion. In order to isolate dynamical regions experimentally, one could simply select a sufficiently dense grid of passive tracers, advect it under the velocity field, and look at the tracer distribution at different times. As time evolves, the snapshots obtained in this fashion provide clues for $t=$ const. cross-sections of dynamical regions. A typical result of such an experiment is shown in Fig. 1. One can make the following observations based on these pictures:

1. Local stretching in the flow appears to happen across coherent structure boundaries. In particular, blobs of particles travel together around such boundaries and then suddenly depart in opposite directions, following dynamically different patterns (see Fig. 2a). In other words, parts of the coherent structure boundaries are responsible for local instabilities in the flow.

2. Thinning and folding appear to happen along coherent structure boundaries. In particular, departing tracer streaks tend to become thinner and thinner

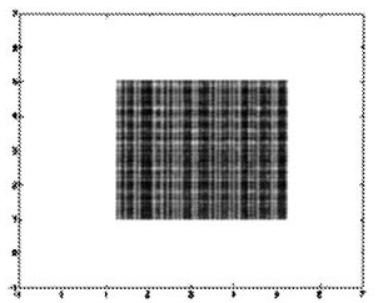

(a)

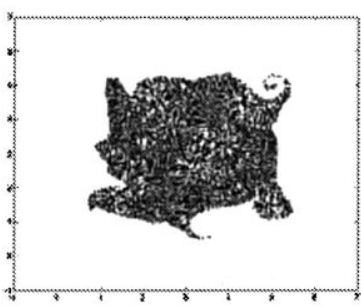

(c)

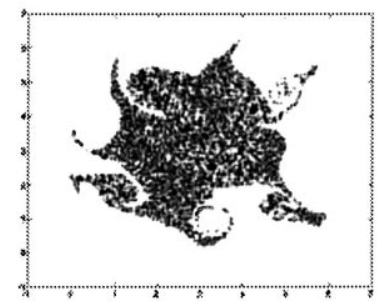

(e)

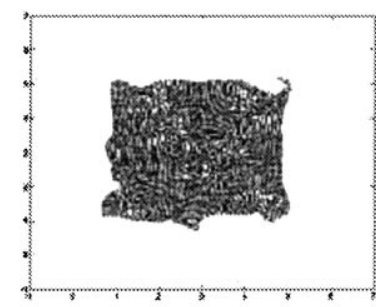

(b)

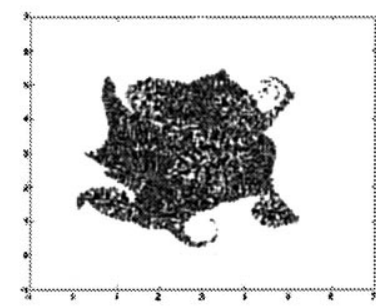

(d)

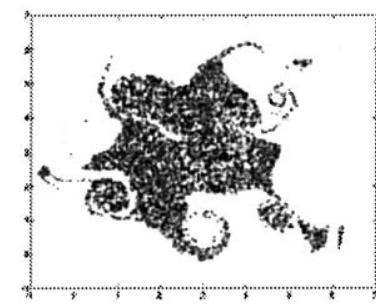

(f)
Fig. 1. Evolution of an initial rectangular set of tracers in two-dimensional barotropic turbulence (see Section 12 for more information).

as they travel along the boundaries. Folding then occurs because of the global curved shape of the boundaries they follow (see Fig. 2b). Therefore,
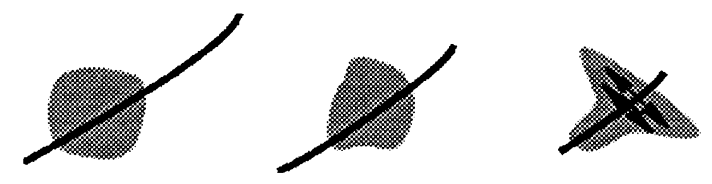

(a)
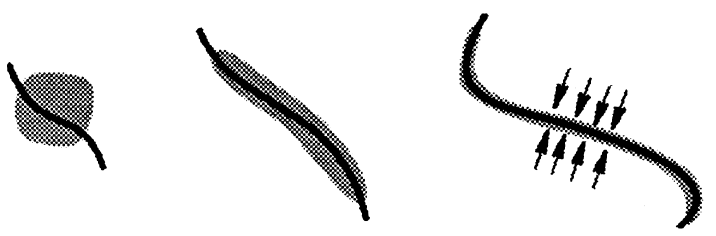

(b)

Fig. 2. The role of coherent structure boundaries in: (a) stretching, (b) folding. 
thinning and folding are due to local stabilities in the flow.

These two observations prompt us to approach coherent structures boundaries as material lines that create smaller tracer scales through stability or instability.

\section{Material lines and surfaces}

Consider a two-dimensional velocity field

$\dot{\mathbf{x}}=\mathbf{u}(\mathbf{x}, t)$

on some finite-time interval $\left[t_{-1}, t_{1}\right]$. We emphasize that the velocity field is only known for finite times, a fact that one has to face in the study of any numerically or experimentally given turbulent velocity field.

In fluid mechanics the particle trajectories $\mathbf{x}(t)$ generated by this velocity fields are called pathlines and are typically visualized as curves on the $\mathbf{x}$ plane. These curves will in general be self-intersecting for an unsteady velocity field. A curve of initial conditions, $\Gamma_{0}$, on the $\mathbf{x}$ plane can be advected by the velocity field in time, in which case later images of $\Gamma_{0}$ at time $t$, denoted by $\Gamma_{t}$, are called material lines.

We would like to define a framework in which the stability or instability of a material line can be studied on the finite-time interval $\left[t_{-1}, t_{1}\right]$. To be able to use stability concepts from dynamical systems theory, we shall mostly think of the fluid particle motions $\mathbf{x}\left(t ; t_{0}, \mathbf{x}_{0}\right)$ induced by the velocity field as trajectories in the space of the variables $(\mathbf{x}, t)$, which start from the point $\mathbf{x}_{0}$ at time $t_{0} \in\left[t_{-1}, t_{1}\right]$. Trajectories in this extended phase space do not intersect and hence the different qualitative behaviors of different sets of initial conditions are easier to visualize. The map

$\mathbf{F}_{t_{0}}^{\tau}: \mathbf{x}_{0} \mapsto \mathbf{x}\left(t_{0}+\tau ; t_{0}, \mathbf{x}_{0}\right)$

relates initial conditions at $t_{0}$ to their later position at time $t_{0}+\tau$. For simplicity, we shall refer to this map as the flow map. ${ }^{1}$ There is an associated extended flow

\footnotetext{
${ }^{1}$ Note, however, that this map is not a flow in the mathematical sense of the word.
}

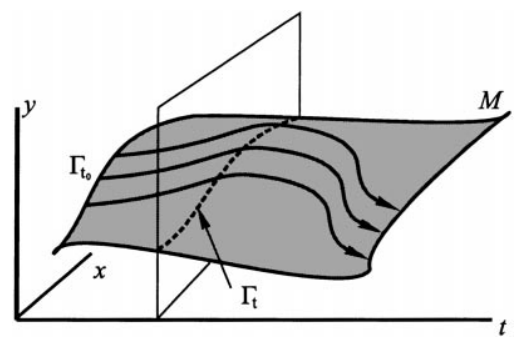

Fig. 3. The evolving material curve $\Gamma_{t}$ spans out a material surface $M$ in the extended phase space.

map defined on the extended phase space as

$\mathcal{F}^{\tau}:\left(\begin{array}{c}\mathbf{x}_{0} \\ t_{0}\end{array}\right) \mapsto\left(\begin{array}{c}\mathbf{x}\left(t_{0}+\tau ; t_{0}, \mathbf{x}_{0}\right) \\ t_{0}+\tau\end{array}\right)$.

A deforming material line $\Gamma_{t}$ spans a two-dimensional surface $M$ in the $(\mathbf{x}, t)$ space, which we call a material surface (see Fig. 3). The dynamical stability of the material line $\Gamma_{t}$ can then be studied in terms of the stability of $M$ in the extended phase space.

In order to describe the stability of $M$, we shall need to linearize the extended flow map $\mathcal{F}^{\tau}$ along $M$. First, recall that the linearized flow map along a particle path $\mathbf{x}\left(t_{0}+\tau ; t_{0}, \mathbf{x}_{0}\right)$ is given by $\nabla \mathbf{F}_{t_{0}}^{\tau}\left(\mathbf{x}_{0}\right)$, which is called the deformation gradient in continuum mechanics. It describes the evolution of infinitesimal perturbations along the trajectory starting from $\mathbf{x}_{0}$ : A small disturbance $\mathbf{x}_{0}+s \mathbf{e}_{0}$ of the initial condition will be carried by the velocity field to the point $\mathbf{x}\left(t_{0}+\tau ; t_{0}, \mathbf{x}_{0}\right)+s \nabla \mathbf{F}_{t_{0}}^{\tau}\left(\mathbf{x}_{0}\right) \mathbf{e}_{0}+\mathcal{O}\left(s^{2}\right)$. Using the deformation gradient, one can compute the linearized extended flow map $D \mathcal{F}^{\tau}$, given by the $3 \times 3$ matrix

$D \mathcal{F}^{\tau}=\left(\begin{array}{cc}\nabla \mathbf{F}_{t_{0}}^{\tau} & \partial_{t_{0}} \mathbf{F}_{t_{0}}^{\tau} \\ \mathbf{O} & 1\end{array}\right)$

Here $\partial_{t_{0}} \mathbf{F}_{t_{0}}^{\tau}$ is a two-dimensional column vector and $\mathbf{O}$ is a two-dimensional row vector of zeros. We note that $\partial_{t_{0}} \mathbf{F}_{t_{0}}^{\tau}=\mathbf{u}\left(\mathbf{x}\left(t_{0}+\tau\right), t_{0}+\tau\right)-\mathbf{u}\left(\mathbf{x}_{0}, t_{0}\right)$.

\section{Stable and unstable material surfaces}

We are interested in locating material lines that are responsible for creating the type of local instability in tracer mixing that we described in Section 2. This instability manifests itself in directions normal to a 
material line $\Gamma_{t}$, and for this reason we shall not be concerned with instabilities in the direction tangential to material lines. Generically, such a "normal" instability will be captured by appropriate linearization along the corresponding material surface $M$. In view of this, we want to call $M$ unstable if there is a connected time interval $I_{\mathrm{u}}=\left[t_{-}, t_{+}\right] \subset\left[t_{-1}, t_{1}\right]$, such that during $I_{\mathrm{u}}$ all nearby fluid trajectories separate from $M$ at an exponential rate.

While characterizing the instability of $M$ for infinite times is well understood in dynamical systems (see, e.g., [15]), one has to be careful in phrasing a reasonable concept of instability on a finite-time interval. If the distance of a fluid trajectory $\mathbf{p}(t)=(\mathbf{x}(t), t)$ from $M$ at $t_{-}$and $t_{+}$is such that

$\operatorname{dist}\left(\mathbf{p}\left(t_{-}\right), M\right)<\operatorname{dist}\left(\mathbf{p}\left(t_{+}\right), M\right)$,

then one can always find an exponent $\lambda_{\mathrm{u}}>0$ such that

$\operatorname{dist}\left(\mathbf{p}\left(t_{+}\right), M\right) \geq \operatorname{dist}\left(\mathbf{p}\left(t_{-}\right), M\right) \mathrm{e}^{\lambda_{\mathrm{u}}\left(t_{+}-t_{-}\right)}$.

However, this relation by itself says nothing about the stability type of $M$ on the interval $I_{\mathrm{u}}$ : It might have been stable for the most part, or might have been changing its stability, as shown in Fig. 4. Therefore, to capture a consistent unstable behavior for $M$ on the interval $I_{\mathrm{u}}$, it is not enough to require a condition of the type (2) to hold for all nearby initial conditions. Rather, to obtain a reasonable concept of finite-time instability, one should require exponential separation from $M$ on arbitrarily short time intervals within the instability interval $I_{\mathrm{u}}$. In particular, we call $M$ an $u n$ stable material surface on the time interval $I_{\mathrm{u}}$ if there is a positive exponent $\lambda_{\mathrm{u}}$ such that for any close enough

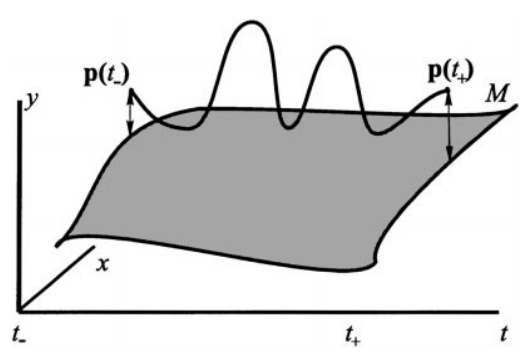

Fig. 4. Increased distance from a material surface at $t_{+}$does not imply sustained linear instability on the time interval $\left[t_{-}, t_{+}\right]$.

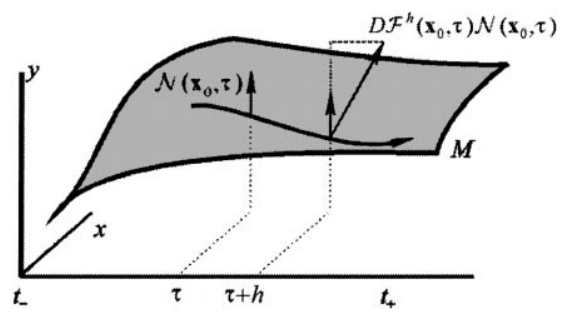

Fig. 5. The geometry of the linear instability condition for a material surface.

(i.e., "infinitesimally close") initial condition $\mathbf{p}(\tau)=$ $(\mathbf{x}(\tau), \tau)$ and for any small time step $h>0$ we have

$\operatorname{dist}(\mathbf{p}(\tau+h), M) \geq \operatorname{dist}(\mathbf{p}(\tau), M) \mathrm{e}^{\lambda_{\mathrm{u}} h}$,

as long as $\tau$ and $\tau+h$ are both taken from $I_{\mathrm{u}}$.

For (3) to hold, one simply has to require that the normal components of vectors initially orthogonal to $M$ in the extended phase space should grow exponentially, obeying a uniform lower bound for the exponent. In particular, if $\mathcal{N}(\mathbf{p}, t)$ is a unit normal to $M$ at the point $(\mathbf{p}, t)$ in the extended phase space, then $M$ is an unstable material surface over $I_{\mathrm{u}}$ if for all $\tau, \tau+h \in$ $I_{\mathrm{u}}$ and for all initial conditions $\left(\mathbf{x}_{0}, \tau\right) \in M$, we have

$\left|\mathcal{N}(\mathbf{x}(\tau+h), \tau+h) \cdot D \mathcal{F}^{h}\left(\mathbf{x}_{0}\right) \mathcal{N}\left(\mathbf{x}_{0}, \tau\right)\right| \geq \mathrm{e}^{\lambda_{\mathrm{u}} h}$,

where $\mathbf{x}(\tau+h)$ is the trajectory at time $\tau+h$ that started from $\mathbf{x}_{0}$ at time $\tau$, and "." refers to the scalar product of the vectors (see Fig. 5).

As shown in Fig. 6, individual fluid trajectories on $M$ enter the unstable region of $M$ at some time within the interval $I_{\mathrm{u}}$. If, in addition, the flow is incompressible, trajectories must converge to each other on $M$

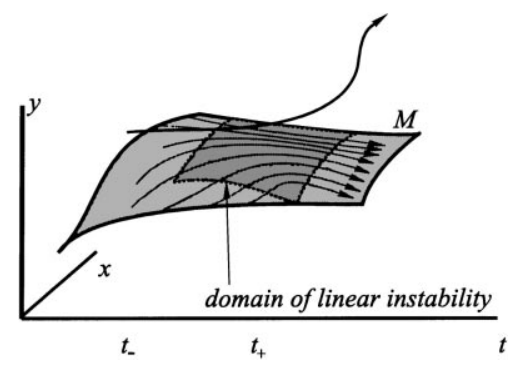

Fig. 6. An unstable material surface $M$ over the time interval $I_{\mathrm{u}}=\left[t_{-}, t_{+}\right]$. 
within the domain of linear instability in order to ensure area preservation for the linearized flow map $\nabla \mathbf{F}_{t_{0}}^{\tau}$ (see Appendix A).

We should note here that a material surface with a positive finite-time Lyapunov exponent on any subinterval of $I_{\mathrm{u}}$ is in general not an unstable material surface. The simplest example is given by the steady linear velocity field $\dot{x}_{1}=-x_{1}, \dot{x}_{2}=x_{2}$. The material surface $M=\left\{x_{1}=0\right\}$ is not an unstable material surface in the sense of (4), as it attracts trajectories exponentially. Yet, for any trajectory in $M$, one of the finite-time Lyapunov exponents is +1 .

Recalling the third observation of Section 2, we also need to define stable material surfaces. We propose that these are the surfaces responsible for the quick thinning of fluid streaks after their departure from an unstable material surface. They also tend to guide the departing streaks through global folding. We therefore define a stable material surface $N$ as a smooth material surface that is unstable in backward time, in the sense of (4), with instability interval $I_{\mathrm{s}}$.

An unstable material line with instability interval $I_{\mathrm{u}}$ is just a curve $\Gamma_{t}$ which generates an unstable material surface in the extended phase space with instability interval $I_{\mathrm{u}}$. A similar definition can be used to defined stable material lines.

We shall refer to unstable and stable material lines (surfaces) as hyperbolic material lines (surfaces), motivated by the terminology used in the theory of infinite-time invariant manifolds. Accordingly, the corresponding intervals $I_{\mathrm{u}}$ and $I_{\mathrm{S}}$ will be referred to as hyperbolicity intervals.

\section{Local nonuniqueness of hyperbolic material lines}

Assume now that a material surface $M$ is unstable on the interval $I_{\mathrm{u}}$. By the continuous dependence of the right-hand side of (4) on all its arguments, close enough material surfaces will also be unstable for a while. They, however, have to be very close to $M$ in order to be unstable in the sense of (4) on the whole time interval $I_{\mathrm{u}}$. The reason is that the instability of $M$ pushes them away at an exponential rate from the neighborhood in which the continuity argument holds. It can be deduced from the finite-time invariant manifold results of Haller and Poje [16] that any nearby unstable material surface $M^{\prime}$ with instability interval $I_{\mathrm{u}}$ must obey the distance estimate

$\underset{t \in I_{\mathrm{u}}}{\operatorname{dist}}\left(M, M^{\prime}\right)<C_{0} \mathrm{e}^{-\lambda_{\mathrm{u}}\left(t_{+}-t_{-}\right)}$,

where $C_{0}$ can be selected fixed as long as $t_{1}-t_{0}$ and the fluid domain of interest is bounded. This estimate indicates that, while unstable material surfaces are never unique on a finite-time interval $\left[t_{0}, t_{1}\right]$, the degree of their nonuniqueness is exponentially small with respect to the product of the instability exponent $\lambda_{\mathrm{u}}$ and the length of the instability interval $I_{\mathrm{u}} \cdot{ }^{2}$ As a result, material lines that are hyperbolic for long enough time intervals will appear to be locally unique up to numerically unresolvable errors. This gives a strong motivation to use stable and unstable material surfaces in the definition of coherent structure boundaries.

\section{Definition of coherent structure boundaries}

For any initial condition $\mathbf{x}_{0}$ at time $t_{0} \in\left[t_{-1}, t_{1}\right]$, consider the maximal open set, $\mathcal{I}_{\mathrm{u}}\left(\mathbf{x}_{0}\right)$, within $\left[t_{0}, t_{1}\right]$ on which the instability condition (4) holds. Note that $\mathcal{I}_{\mathrm{u}}\left(\mathbf{x}_{0}\right)$ is a set of connected open intervals, with each of its connected components satisfying (4) with an appropriate exponent $\lambda_{\mathrm{u}}$. We define the (total linear) instability time $T_{\mathrm{u}}\left(\mathbf{x}_{0}, t_{0}\right)$ associated with $\mathbf{x}_{0}$ over the time interval $\left[t_{0}, t_{1}\right]$ as

$T_{\mathrm{u}}\left(\mathbf{x}_{0}, t_{0}\right)=\frac{1}{t_{1}-t_{0}} \int_{\mathcal{I}_{\mathrm{u}}\left(\mathbf{x}_{0}\right)} \mathrm{d} t$.

Similarly, $\mathcal{I}_{\mathrm{S}}\left(\mathbf{x}_{0}\right)$ will denote the maximal open set in $\left[t_{0}, t_{-1}\right]$ on which (4) is satisfied in backward time.

\footnotetext{
${ }^{2}$ The continuity argument we used for the nonuniqueness of $M$ breaks down if $I_{\mathrm{u}}$ is an unbounded interval. For instance, periodic velocity fields of the form (1) can be considered as given on infinite time intervals. Any bounded piece of an unstable material surface with instability interval $I_{\mathrm{u}}=[-\infty,+\infty]$ is then a normally hyperbolic invariant manifold, which is locally unique by classic results from dynamical systems theory (see, e.g., [15]).
} 


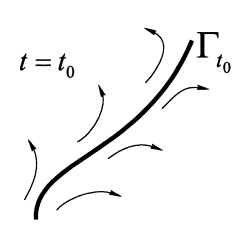

(a) (b)

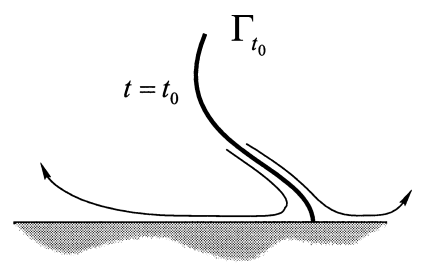

Fig. 7. Coherent structure boundaries: (a) maximizing $T_{\mathrm{u}}$, (b) minimizing $T_{\mathrm{u}}$.

Then the (total linear) stability time $T_{\mathrm{S}}\left(\mathbf{x}_{0}, t_{0}\right)$ associated with $\mathbf{x}_{0}$ over the backward time interval $\left[t_{0}, t_{-1}\right]$ is

$T_{\mathrm{S}}\left(\mathbf{x}_{0}, t_{0}\right)=\frac{1}{t_{0}-t_{-1}} \int_{\mathcal{I}_{\mathrm{s}}\left(\mathbf{x}_{0}\right)} \mathrm{d} t$.

We call $T_{\mathrm{u}}\left(\mathbf{x}_{0}, t_{0}\right)$ and $T_{\mathrm{s}}\left(\mathbf{x}_{0}, t_{0}\right)$ the hyperbolicity times associated with $\mathbf{x}_{0}$ at $t_{0}$.

Based on our earlier discussion, we propose the following definition: At $t=t_{0}$, coherent structure boundaries are given by stable and unstable material lines along which $T_{\mathrm{S}}$ or $T_{\mathrm{u}}$ attains local extrema. For instance, $T_{\mathrm{u}}$ is said to be locally maximal along a material line if, near this line, we do not find initial conditions with longer instability times.

Material lines locally maximizing $T_{\mathrm{u}}$ keep pushing away particles on both sides for locally the longest times, and hence should indeed be considered as separating lines between two dynamically different regions (see Fig. 7a). For the same reason, material lines maximizing $T_{\mathrm{S}}$ become distinguished in backward time, giving rise to folding in forward time (see Section 2).

For flows near a wall some coherent structure boundaries might be connected to the wall, containing particles that approach the wall in forward time, at least on the time interval of interest. Then, in case of a no-slip boundary condition at the wall, the corresponding dynamical boundary cannot be linearly unstable in its normal direction due to the degeneracy of the flow at the wall (see Fig. 7b). Generically, initial conditions on the coherent structure boundary will admit a shorter $T_{\mathrm{u}}$ value than nearby initial conditions, which will leave a vicinity of the wall later and accumulate more instability time. Therefore, in the generic case, coherent structure boundaries containing separation or reattachment points (with no-slip boundary conditions) can be defined as material lines along which $T_{\mathrm{u}}$ or $T_{\mathrm{S}}$ is locally minimal. At larger distances from the wall, our earlier discussion becomes relevant and the coherent structure boundaries become local maximizers of the $T_{\mathrm{u}}$ or $T_{\mathrm{s}}$ field. This will be described in a companion paper where our methods are applied to a two-dimensional experimental data set (see [11]).

Depending on the maximal length of the available velocity data, the boundaries obtained from the above definition may be thinner or thicker. Once the coherent structure boundaries are found at $t=t_{0}$, later or earlier boundaries can be found by advecting them in forward or backward time. Alternatively, one can change the initial time $t_{0}$ in (5) or (6) to obtain the structure boundaries at $t_{0}$.

We leave it to the reader to verify that for velocity fields given on $\left[t_{-1}, t_{1}\right]=[-\infty, \infty]$, the $t=t_{0}$ slices of the usual stable and unstable manifolds known from dynamical systems are unstable and stable material curves, respectively, in the sense of our definition. For instance, for simple time periodic velocity-fields of the form (1), the coherent structure boundaries we defined above coincide with stable and unstable manifolds of hyperbolic fixed points of the corresponding Poincarémap. However, our approach to these material lines is motivated by their effects on generic tracer patches, rather than by their internal asymptotic dynamics. In particular, from the point of view of mixing, the effect of a classical stable manifold on a tracer patch is instability, while the effect of an unstable manifold is stability.

If there are fluid particles that actually cross a coherent structure boundary, then the boundary will not be a closed curve. For instance, a coherent structure may have a sizable "gate" in it, accounting for the fact that fluid particles are continuously entrained into it. Such structures are, e.g., growing mesoscale eddies in geophysical flows (see, e.g., [39]).

In this paper we shall only deal with open turbulent flows, and hence coherent structure boundaries will always be local maximizers of the $T_{\mathrm{u}}$ or $T_{\mathrm{s}}$ field. However, minimizing boundaries are equally important in several physical applications involving walls. 
For instance, Cohen et al. [11] use these techniques to study experimental data for a turbulent flow around a backward facing step.

\section{Dispersion statistics and structure boundaries}

Our definition of Lagrangian coherent structure boundaries as stable and unstable material lines with locally maximal hyperbolicity times is primarily motivated by observations of the kinematics of turbulent mixing. At the same time, coherent structures in two-dimensional turbulence are often approached from a statistical point of view (see, e.g., [42]). The two main statistical quantities used in their description are the absolute and relative dispersion. The absolute dispersion (or single particle dispersion) is defined as the mean square displacement of individual particles, i.e.,

$$
\begin{aligned}
A^{2}\left(t ; t_{0}\right) & =\left\langle\left|\mathbf{x}_{i}(t)-\mathbf{x}_{i}\left(t_{0}\right)\right|^{2}\right\rangle \\
& =\frac{1}{N} \sum_{i=1}^{N}\left|\mathbf{x}_{i}(t)-\mathbf{x}_{i}\left(t_{0}\right)\right|^{2}
\end{aligned}
$$

with $\mathbf{x}_{i}(t), \ldots, \mathbf{x}_{N}(t)$ denoting an ensemble of particles. In principle, the ensemble average \langle\rangle should be taken at the same spatial position with respect to different realizations of the velocity field. However, in practice one takes a grid of initial conditions and advects it by a given velocity field. Relative dispersion is the mean square displacement of particles with nearby initial conditions:

$D^{2}\left(t ; t_{0}, d_{0}\right)=\frac{1}{N} \sum_{i=1}^{N}\left|\mathbf{x}_{i}(t)-\mathbf{x}_{-i}(t)\right|^{2}$,

where $\mathbf{x}_{i}(t)$ and $\mathbf{x}_{-i}(t)$ are pairs of particles that are initially close, i.e., $\left|\mathbf{x}_{i}\left(t_{0}\right)-\mathbf{x}_{-i}\left(t_{0}\right)\right|=d_{0}$.

While we shall use absolute dispersion to explore the coherent structure boundaries we defined, relative dispersion has a more direct relationship with stable and unstable material lines. Note that if a particle pair is initially located close to an unstable material line $\Gamma_{t_{0}}$, and their initial distance $d_{0}$ is small enough, then $\left|\mathbf{x}_{i}(t)-\mathbf{x}_{-i}(t)\right|$ will experience exponential growth over the instability interval of $\Gamma_{t}$. If $\Gamma_{t}$ is part of a coherent structure boundary, then, by definition, pairs of particles further away from $\Gamma_{t}$ may only experience exponential separation on shorter time scales. By continuity, the exponents governing these short-term exponential separations must be close to the exponent $\lambda_{\mathrm{u}}$ associated with $\Gamma_{t}$. For this reason, the time-dependent scalar field

$d^{2}\left(t, \mathbf{x}_{i}\left(t_{0}\right)\right)=\left|\mathbf{x}_{i}(t)-\mathbf{x}_{-i}(t)\right|^{2}$

should have local maxima along initial conditions $\mathbf{x}_{i}\left(t_{0}\right)$ located on $\Gamma_{t_{0}}$. One therefore expects the relative dispersion, which is just a discrete average of the $d^{2}$-field, to be strongly influenced by the presence of strongly hyperbolic material lines. We shall demonstrate this fact numerically in Section 12. While less obviously related to strongly hyperbolic material lines, the absolute dispersion "density" field

$a^{2}\left(t, \mathbf{x}_{i}\left(t_{0}\right)\right)=\left|\mathbf{x}_{i}(t)-\mathbf{x}_{i}\left(t_{0}\right)\right|^{2}$

is also expected to indicate the presence of Lagrangian coherent structure boundaries due to their global influence on particle mixing. We will also observe this clearly in the numerical simulations of barotropic turbulence presented in Section 12.

\section{Detectability of Lagrangian coherent structure boundaries}

While condition (4) can be adopted as an exact mathematical definition for Lagrangian coherent structure boundaries, it would be rather difficult to verify in practice. The computation of the linearized flow map $D \mathcal{F}_{\tau}^{h}\left(\mathbf{x}_{0}\right)$ for all possible $\tau$ and $h$ values and for all possible choices of material lines is clearly not feasible.

One might obtain clues about the location of strongly stable material lines from $t=$ const. streamline pictures that show instantaneous saddle-type stagnation points with unstable manifolds. If the velocity field varies slowly enough in time, such an unstable manifold in the velocity contour is expected to be close to stable material surfaces. Although phrased differently, this was the underlying 
assumption in several recent studies that were aimed at locating unstable manifolds in geophysical flows (see $[12,16,24,30,43])$. In these studies pieces of instantaneously stable streamlines were advected ("straddled") and their convergence to an underlying unstable manifold in the flow was assumed. Haller and Poje [16] gave analytic conditions under which the existence of a (nonunique) nearby finite-time unstable manifold can indeed be inferred from instantaneous stagnation points. However, they also showed that if these conditions are violated, straddling may lead to "phoney" unstable manifolds.

Poje et al. [40] explored the relevance of finite-time stable and unstable manifolds obtained from straddling to patchiness plots, a statistical edge detection tool suggested by Mezić and Wiggins [29]. They found that on intermediate time scales, plots of the $x$ or $y$ component of average Lagrangian velocities show a visible relationship with such velocity patches. On longer time scales, however, this relationship is necessarily lost.

Recently, Bowman [8] suggested a "finite strain map" technique for locating unstable manifolds without relying on instantaneous stagnation points. In the context of our current discussion, the finite strain map technique can be viewed as a global search for unstable material lines. In Bowman's work these curves are sought as curves of initial conditions for which the total linear separation of nearby tracers is maximal. This is equivalent to searching for local maxima in the relative dispersion density field $d^{2}$ defined in (7). For this reason, finite strain maps are highly relevant in the detection of Lagrangian coherent structure boundaries. In fact, the more recent work of von Hardenberg et al. [19] on mixing in a baroclinic atmosphere model clearly shows that such boundaries are indeed local maximizers of the relative dispersion field. In practice, however, two limitations of this approach arise. First, local maxima in the $d^{2}(t, \mathbf{x})$ field are necessary but not sufficient indicators of the existence of finite-time hyperbolic material lines. For instance, tangentially unstable material lines will also show up as local maxima. As a result, a horizontal nonlinear shear flow can produce sharp local maxima for $d^{2}$ without admitting any stable or unstable material surfaces in the sense of (4). This fact makes it difficult to evaluate the results obtained from finite strain maps. Second, as time evolves, regions of high strain will produce large values for $d^{2}$, which will completely suppress many of its local maxima. In particular, important material lines may simply not show up, become fuzzy, or soon disappear from the time-dependent contour plots of $d^{2}$ (see [17] for examples).

All this suggests a need for an algorithm that reliably extracts coherent structure boundaries from numerical or experimental velocity data. Reasonable requirements for such an algorithm include a solid mathematical foundation, increased accuracy (convergence) as the available time interval is increased, and Galilean invariance (no reliance on stagnation points).

\section{An analytic criterion for coherent structure boundaries}

In this section we present an analytic criterion that can be used to find material lines satisfying the instability requirement (4). This will enable us to locate coherent structure boundaries at any fixed time $t$ by plotting a hyperbolicity time distribution at $t$ and looking for curves along which hyperbolicity times are locally maximal.

The criterion we give below is a sufficient condition for the existence of stable and unstable material surfaces, and is based on recent advances in dynamical systems theory. In particular, our criterion will build on some uniform finite-time hyperbolicity results for two-dimensional vector fields from Haller [17]. For simplicity, throughout this section we assume that

$\nabla \cdot \mathbf{u}=0$

i.e., the underlying fluid is incompressible. We briefly discuss the case of compressible flows after the main result.

Assume that a fluid particle path $\mathbf{x}(t)$ generated by the velocity field (1) is known. The Jacobian of the velocity field along $\mathbf{x}(t)$ is given by the time-dependent matrix $\nabla \mathbf{u}(\mathbf{x}(t), t)$, where $\nabla$ denotes differentiation with respect to $\mathbf{x}$. We assume that on a closed time 
interval $I_{\mathrm{u}} \subset\left[t_{0}, t_{1}\right]$, the relation $\operatorname{det} \nabla \mathbf{u}(\mathbf{x}(t), t)<0$ holds, i.e., $\nabla \mathbf{u}(\mathbf{x}(t), t)$ has real eigenvalues $-\lambda(t)<$ $0<\lambda(t)$ (here we used (9)). We shall use the quantity

$\lambda_{\min }=\min _{t \in I_{\mathrm{u}}} \lambda(t)$.

The eigenvectors $\mathbf{e}_{1}(t)$ and $\mathbf{e}_{2}(t)$ correspond to $-\lambda(t)$ and $\lambda(t)$, selected in a way so that they vary smoothly in $t$. We also assume that they are normalized so that $\left|\mathbf{e}_{k}(t)\right|=1$. We denote the angle between $\mathbf{e}_{1}(t)$ and $\mathbf{e}_{2}(t)$ by $\kappa(t)$ and note that $\sin \kappa(t) \neq 0$. For matrices $\mathbf{A} \in \mathbb{R}^{2 \times 2}$, we shall use the norm $|\mathbf{A}|=$ $\left(\sum_{i, j=1}^{2}\left|A_{i j}\right|^{2}\right)^{1 / 2}$. In this notation, the matrix of eigenvectors $\mathbf{T}(t)=\left[\mathbf{e}_{1}(t), \mathbf{e}_{2}(t)\right]$ satisfies $|\mathbf{T}|=\sqrt{2}$ and $|\operatorname{det} \mathbf{T}|=|\sin \kappa(t)|$. We shall also need the quantities

$\alpha=\min _{t \in I_{\mathrm{u}}}|\operatorname{det} \mathbf{T}|, \quad \beta=\max _{\left.t \in I_{\mathrm{u}}\right]}|\dot{\mathbf{T}}(t)|$,

which are upper bounds on the norm of $\mathbf{T}^{-1} / \sqrt{2}$ and $\dot{\mathbf{T}}$, respectively. Recall that $\alpha$ is never zero by definition.

Using these quantities we have the following result.

Theorem 1. Suppose that for a fluid trajectory $\mathbf{x}(t)$ of the velocity field (1) we have

$\operatorname{det} \nabla \mathbf{u}(\mathbf{x}(t), t)<0, \quad \lambda_{\min }>(2+\sqrt{2}) \frac{\beta}{\alpha}$.

Then $\mathbf{x}(t)$ is contained in a material line $\Gamma_{t}$ which is unstable on the time interval $I_{\mathrm{u}}$. Furthermore, the instability exponent in (4) can be estimated as

$\lambda_{\mathrm{u}}=\lambda_{\min }-\mathcal{O}\left(\frac{\beta}{\alpha}\right)$.

Proof. See Appendix A.

Several important remarks are in order:

1. Physically, Theorem 1 requires that typical Lagrangian velocities near an unstable material line should be larger than the deformation rate of the curve itself. This is a very natural requirement for a coherent structure boundary, since randomly picked material lines in a turbulent flow field will deform at a speed dictated by individual particle velocities.
2. The criterion (12) is Galilean invariant, i.e., it will be satisfied for the same material line even after a change of coordinates $\mathbf{x}^{\prime}=\mathbf{Q x}+\mathbf{v}_{0} t$, where $\mathbf{Q}$ is a proper orthogonal, $2 \times 2$ matrix and $\mathbf{v}_{0}$ is a constant velocity. In fact, our results are invariant under the more general set of transformations $\mathbf{x}^{\prime}=$ $\mathbf{Q}(t) \mathbf{x}+\mathbf{v}(t)$, where $\mathbf{v}(t)$ is an arbitrary function of time and the matrix $\mathbf{Q}$ is not varying faster in time than $\mathcal{O}(\beta / \alpha)$.

3. We stress that despite its simple appearance, the criterion (12) is nonlinear in nature. This can be seen by noting, that computing the quantity $\beta$ one needs to evaluate second derivatives of the velocity field. Furthermore, (12) needs to be computed along fluid trajectories that satisfy the full nonlinear Eq. (1).

4. Stable material lines satisfy the same criterion. In order to find them numerically, one has to verify (12) in backward time.

5. A result analogous to Theorem 1 can be obtained for compressible flows by substituting the conditions (A.1)-(A.4) of Appendix A for (12). For weakly incompressible flows, however, (12) and (A.1)-(A.4) seem to produce indistinguishable results.

6. Theorem 1 provides a sufficient condition for the existence of hyperbolic material lines. If one is only interested in locating coherent structures whose "bulk" deformation rate is slower than typical nearby Lagrangian particle speeds, the conditions of the theorem also become necessary (see [17]).

\section{Vortex boundaries and the Okubo-Weiss criterion}

The first condition of Theorem 1 provides a relationship between the Lagrangian coherent structure boundaries we defined and the well-known Okubo-Weiss criterion for a rough partition of two-dimensional turbulence (see, e.g., $[27,33,48,49])$. In particular, letting

$Q(\mathbf{x}, t)=s^{2}(\mathbf{x}, t)-\omega^{2}(\mathbf{x}, t)$, 
where

$\omega^{2}=\left(\partial_{x} u_{y}-\partial_{y} u_{x}\right)^{2}$,

$s^{2}=\left(\partial_{x} u_{x}-\partial_{y} u_{y}\right)^{2}+\left(\partial_{y} u_{x}+\partial_{x} u_{y}\right)^{2}$

are the squared vorticity and strain, the regions instantaneously dominated by strain satisfy

$Q(\mathbf{x}, t)>0 \Leftrightarrow \operatorname{det} \nabla \mathbf{u}(\mathbf{x}, t)<0$.

According to the Okubo-Weiss principle, these regions are considered hyperbolic. Since the material lines satisfying (12) turn out to be uniformly hyperbolic over the interval $I_{\mathrm{u}}$ (see Appendix A), our result can be considered as a mathematically rigorous connection between instantaneous hyperbolic regions obtained from the Okubo-Weiss partition and actual hyperbolic particle behavior. It is consistent with the conclusion of Basdevant and Philipovitch [6], who pointed out that the Okubo-Weiss criterion may only identify elliptic and hyperbolic regions correctly near the cores of vortices and hyperbolic stagnation points. Furthermore, at least for hyperbolic regions, it extends the second-order correction to the Okubo-Weiss criterion by Hua and Klein [21] to an exact, "all-order" correction.

At the same time, Theorem 1 underlines the fact that vortex boundaries should not be defined as sets satisfying $Q(\mathbf{x}, t)=0$. While this criterion was used as a "working definition" in several studies, most authors have noted its shortcomings (see [42] for an overview). Theorem 1 shows that the most important parts of vortex boundaries, i.e., the parts that are linearly stable or unstable, actually lie in regions where $Q(\mathbf{x}, t)>0$. The remaining parts of the boundaries might also remain in $Q(\mathbf{x}, t)>0$ regions, or might also cross to $Q(\mathbf{x}, t)<0$ regions. In our simulations described in Section 12 , they tended to remain in the $Q(\mathbf{x}, t)>0$ region.

\section{Detecting coherent structures numerically}

The statement of Theorem 1 leads to the following procedure for the extraction of Lagrangian coherent structure boundaries from the velocity field (1):
1. Consider a grid of initial conditions at $t=t_{0} \in$ $\left[t_{-1}, t_{1}\right]$.

2. Integrate each initial condition $\mathbf{x}_{0}$ forward in time up to $t_{1}$, and determine the total instability time $T_{\mathrm{u}}\left(\mathbf{x}_{0}, t_{0}\right)$ defined in (5).

3. Plot the scalar field $T_{\mathrm{u}}\left(\mathbf{x}_{0}, t_{0}\right)$ and note that the $t=t_{0}$ slice of any coherent structure boundary consisting of an unstable material line (in the sense of (4)) will appear as a curve or thin strip which locally maximizes the $T_{\mathrm{u}}\left(\mathbf{x}_{0}, t_{0}\right)$ field (cf. Section 6).

4. Similarly, calculating $T_{\mathrm{S}}\left(\mathbf{x}_{0}, t_{0}\right)$, defined in (5), in backward time on the time interval $\left[t_{0}, t_{-1}\right]$ will produce $t=t_{0}$ sections of coherent structure boundaries that consist of stable material lines.

5. Allowing for arbitrary positive $\lambda_{\min }$ values in the above numerical algorithm, one searches for any possible coherent structure boundary. Selecting a positive threshold for $\lambda_{\text {min }}$ will serve as a filter; only coherent structure boundaries with approximate stability/instability exponents above this threshold will be located (cf. formula (13)). This enables one to isolate structures with a more prominent role in tracer mixing.

The curves (or thin strips) obtained from steps 3 and 4 are the finest approximations for Lagrangian coherent structure boundaries, given the length of the available velocity data in time. Since a separation of Eulerian and Lagrangian time scales is a frequent (if not essential) property of most robust coherent structures in turbulence, by Remark 6 of Section 9, the algorithm we propose above will locate all robust enough structures in a given flow field.

For the above calculations, the Jacobian $\nabla \mathbf{u}(\mathbf{x}(t), t)$ along the trajectory $\mathbf{x}(t)$ needs to be interpolated. This, along with the numerical imprecisions arising from obtaining $\mathbf{x}(t)$, will introduce noise into the numerical procedure described above. In our experiments (to be described below), the results were robust and seemed independent of the numerical differentiation scheme used. At the same time, spatially more complex flows will require a more careful numerical implementation of the calculation of $\nabla \mathbf{u}(\mathbf{x}(t), t)$. 


\section{Numerical experiments on barotropic turbulence}

\subsection{The numerical model}

In order to test the algorithm we proposed in Section 11, we consider the quasigeostrophic vorticity equation

$\frac{\partial q}{\partial t}+[\psi, q]=-v_{4} \nabla^{4} q$

with hyperviscosity $v_{4}=5 \times 10^{-7}$. The quasigeostrophic potential vorticity $q$ is defined as $q=$ $\nabla^{2} \psi-\gamma^{2} \psi$, with $\psi(x, y, t)$ denoting the nondimensionalized free surface (which acts as a stream function), $\nabla^{2}=\partial_{x}^{2}+\partial_{y}^{2}$, and $\gamma$ is the scaled inverse of the Rossby deformation radius. The above definition for $q$ assumes flat bottom topography. Selecting
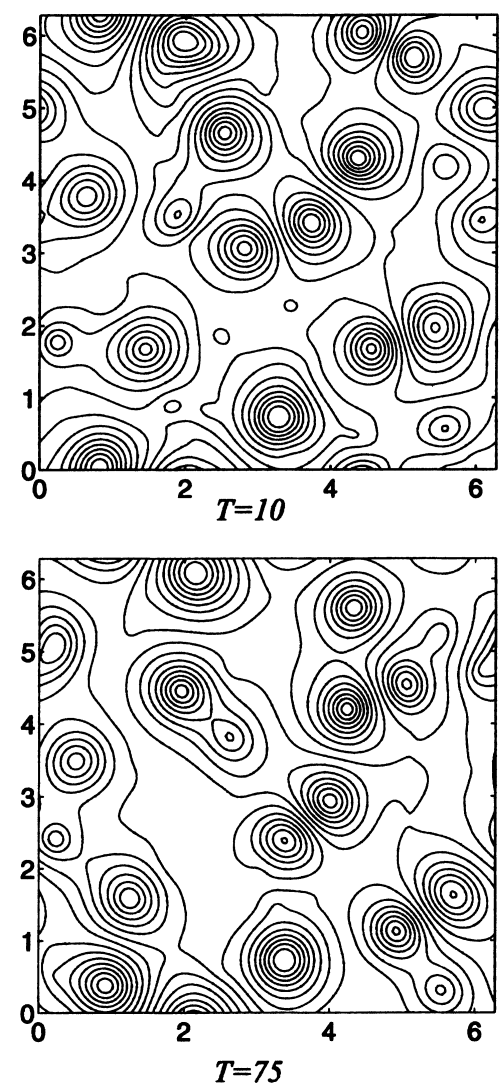

a finite Rossby deformation radius with $\gamma=10$, we aim to produce relatively robust coherent structures, following the approach of Provenzale et al. [41].

Eq. (14) is solved on the square domain $(0,2 \pi)^{2}$, with $128 \times 128$ resolution and with a random Gaussian distribution of vorticity, using the pseudo-spectral code employed in [41]. We started our analysis after robust coherent structures emerged in the flow. Representative contours of the evolution of the studied $q$ distribution are shown in Fig. 8. The $128 \times 128$ velocity field resolution can be considered moderately dense, and was selected to obtain velocity fields on relatively long times with reasonable precision. Snapshots of the evolution of a $256 \times 256$ set of initial tracers were shown in Fig. 1, obtained from a cubic spline interpolation of the velocity field in space and time. For particle tracking, we used the code VFTOOL (see [30]).
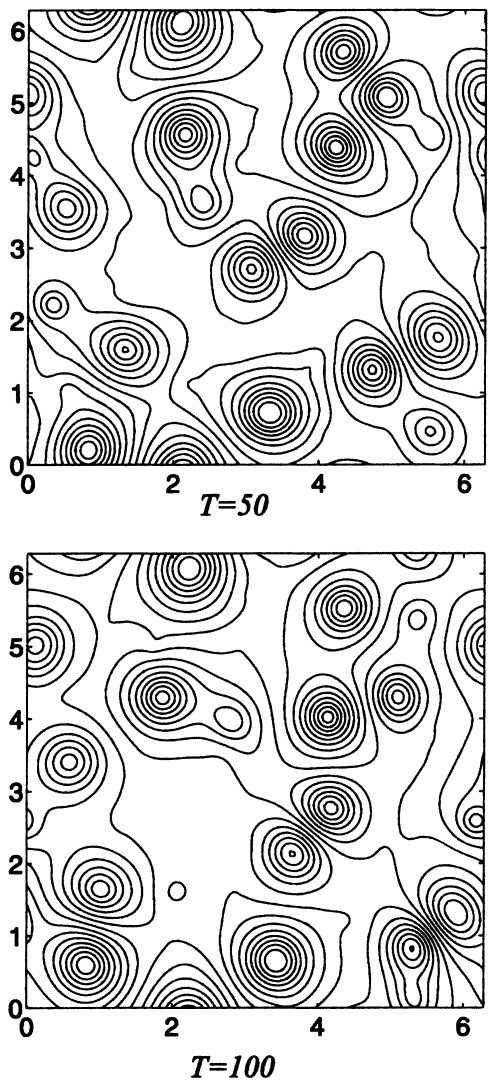

Fig. 8. Potential vorticity contours showing the evolution of vortices. 


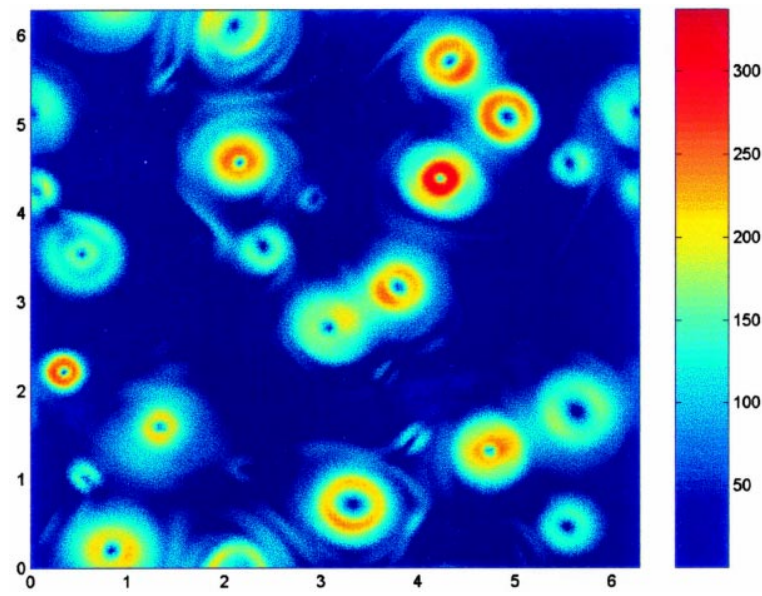

Fig. 9. The magnitude of the potential vorticity gradient at $t_{0}=50$. Local maxima are usually considered as instantaneous vortex boundaries in an Eulerian sense.

\subsection{Eulerian coherent structure description}

Fig. 9 shows the field $|\nabla q(\mathbf{x}, 50)|$, whose maxima are commonly considered in the literature as instantaneous vortex boundaries in the Eulerian sense (cf. Section 1 for references). The corresponding Okubo-Weiss partition (cf. Section 10) to instantaneously hyperbolic and elliptic regions is shown for the same time slice in Fig. 10.

Both Eulerian plots give an instantaneous partition of the physical space and suggest elliptic patches of

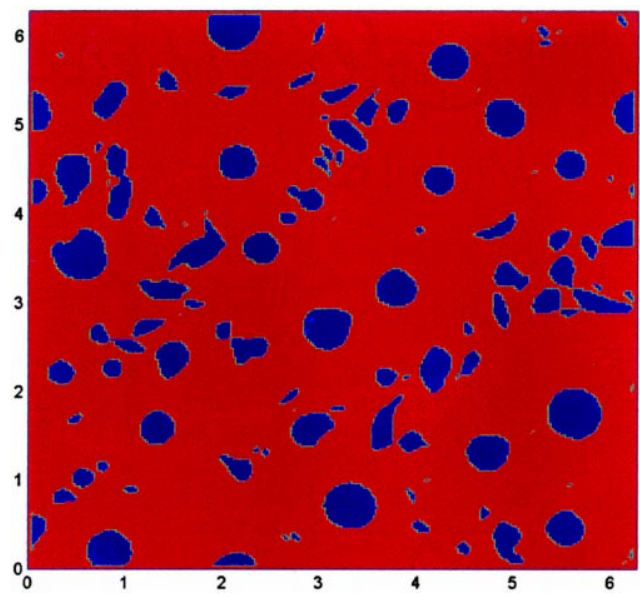

Fig. 10. The Okubo-Weiss partition of the velocity field into hyperbolic (red) and elliptic (blue) regions at $t=50$.

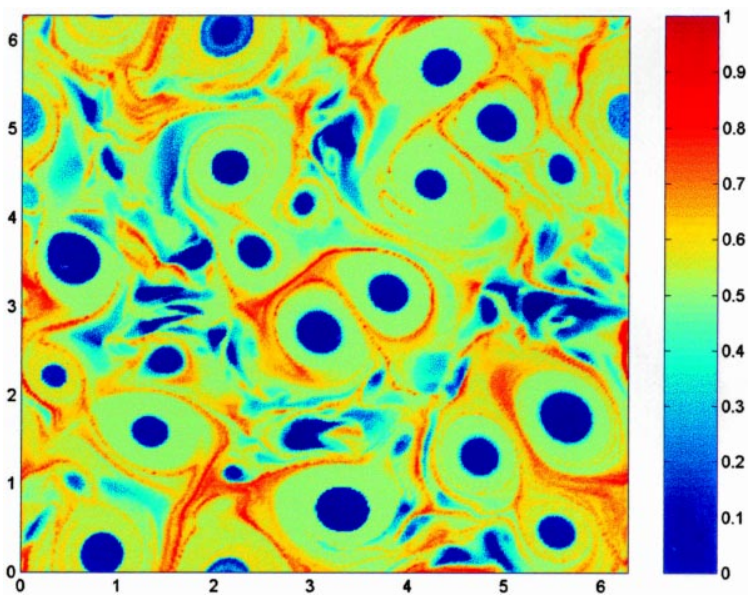

Fig. 11. The instability time field $T_{\mathrm{u}}\left(\mathbf{x}_{0}, 50\right)$. The maximizing curves are repelling Lagrangian coherent structure boundaries (finite-time stable manifolds).

initial conditions that are likely to preserve their coherence for longer times. At the same time, these plots do not give direct clues about mixing and transport in the flow. In particular, they do not reveal the structures responsible for stretching and folding, or the global geometry of mixing within the hyperbolic "background" turbulence.

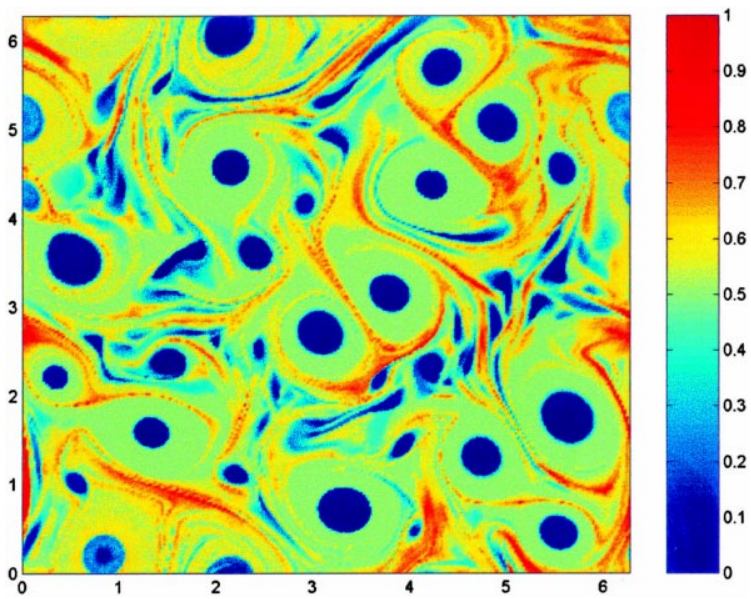

Fig. 12. The stability time field $T_{\mathrm{s}}\left(\mathbf{x}_{0}, 50\right)$. The maximizing curves are attracting Lagrangian coherent structure boundaries (finite-time unstable manifolds) 


\subsection{Lagrangian coherent structures}

In order to obtain more insight into the geometry of mixing in the numerical model (14), we implemented the results of Theorem 1 in a numerical algorithm following the steps described in Section 11. The Jacobian of the velocity field along particle trajectories is obtained from cubic spatial interpolation and centered finite differences. In our calculations we only used the velocity field at increments of $10 h$, where $h=0.1$ is the time step used in the turbulence solver. We chose not to use all the available velocity data in order to show the robustness of our methods and reduce computation time. The sharpness of the results below gives a strong argument for the applicability of this type of analysis to moderately resolved experimental data sets (see also [11] for another example).

Fig. 11 shows the results of our Lagrangian analysis, the total instability time field $T_{\mathrm{u}}\left(\mathbf{x}_{0}, t_{0}\right)$, with the calculation performed on the interval $\left[t_{0}, t_{1}\right]=$ [50,99]. Fig. 12 shows the field $T_{\mathrm{s}}\left(\mathbf{x}_{0}, t_{0}\right)$ calculated on $\left[t_{0}, t_{-1}\right]=[50,10]$. It is worth noting that $T_{\mathrm{u}}\left(\mathbf{x}_{0}, t\right)$ and $T_{\mathrm{S}}\left(\mathbf{x}_{0}, t\right)$ both coincide with the Okubo-Weiss plot of Fig. 10 in the limit $t \rightarrow 50$. Practically speaking, the stability and instability-time plots emerge from the Okubo-Weiss plot through a gradual loss of high (red) values to lower (blue) values. Initial conditions that are truly finite-time hyperbolic for long times stay highlighted, while the ones that were only instantaneously hyperbolic in the Okubo-Weiss sense fade away. The ones that stay truly hyperbolic for locally the longest time then emerge as coherent structure boundaries.

The details provided by Figs. 11 and 12 are striking and reveal unexpected global connections between structures in the background turbulence. The coherent structure boundaries obtained in this fashion are dynamically exact, i.e., reflect the true organizing centers of finite-time Lagrangian mixing.

To extract the structure boundaries themselves, in Fig. 13 we highlighted all important attracting material lines at $t_{0}=50$ by taking the gradient of the field $T_{\mathrm{S}}\left(\mathbf{x}_{0}, 50\right){ }^{3}$ The material lines obtained in this fash-

\footnotetext{
${ }^{3}$ The gradient picture renders the Lagrangian structure boundaries quite efficiently since they all correspond to cusp-type maxima in the hyperbolicity time field.
}

ion are the underlying cause for folding in the flow: In accordance with our discussion in Section 2, a fluid blob will fold at some point at an exponential rate if it intersects one of the curves in Fig. 13 at $t_{0}=50$. This figure underlines the complexity of turbulent mixing compared to simple models of chaotic advection, where typically one or two such curves ("infinite-time" unstable manifolds) are present.

While our primary concern has been the isolation of Lagrangian coherent structure boundaries, we have also obtained sharp results for vortex edges. Based on our analysis, vortex edges can be defined as boundaries of regions of particles that stay away from hyperbolic material surfaces on the whole time interval $\left[t_{0}, t_{1}\right]$. A similar definition can be used to isolate "elliptic patches" in background turbulence. Both vortex edges and the boundaries of small elliptic (Lagrangian) patches are clearly visible as closed curves in Fig. 13. They offer a sharper vortex edge definition than that obtained from the plot of $|\nabla q|$ in Fig. 9.

A final remark about Fig. 13 is that as $t \rightarrow \infty$, the distinguished material lines gradually fill up the whole background region outside coherent vortices, since the hyperbolicity times accrued by all initial conditions in this region tend to infinity. However, the focus of this paper is finite-time mixing, in which case one generically obtains local maximizers in hyperbolicity time plots. The reason is that most particles will violate the second condition in (12) from time to time, even if they satisfy the first condition for all times considered.

In the above calculations all unstable material lines were detected (with $\lambda_{\text {min }}>0$ ), including those in the background turbulent field. As we remarked earlier, it also makes sense to compute hyperbolicity times only for tracers yielding $\lambda_{\min }$-values over a given threshold. In this fashion, one can gradually filter out material surfaces from the background turbulent field. In Fig. 14 we selected the hyperbolicity threshold $\lambda_{\min }=1.5$, and only considered initial conditions which satisfied $\lambda(t)>\lambda_{\text {min }}$. This filtering procedure gives a quantitative tool to compare the Lagrangian "strength" of coexisting coherent structures. 


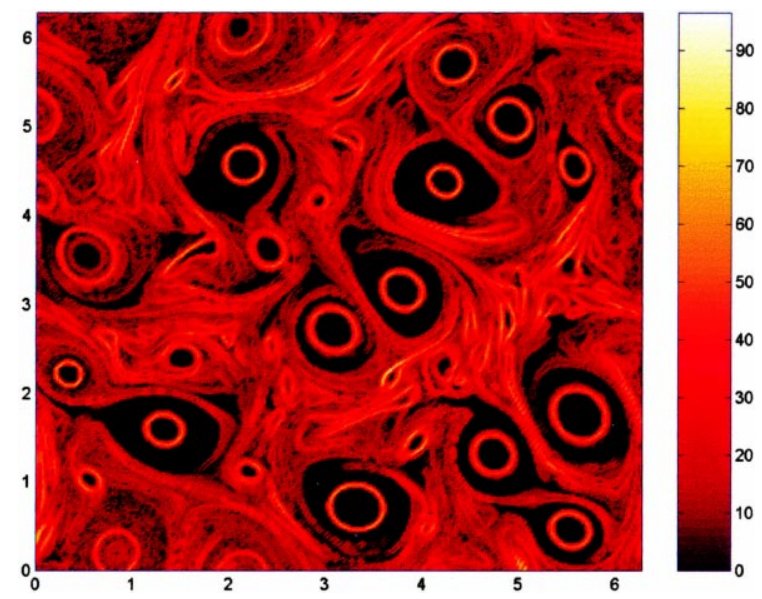

Fig. 13. Attracting Lagrangian coherent structure boundaries (finite-time unstable manifolds) at $t_{0}=50$. These material lines are responsible for the folding of fluid blobs.

\subsection{Comparison with Lagrangian statistics}

To demonstrate the relevance of the Lagrangian structure boundaries to absolute and relative dispersion statistics, we chose to compute the forward-time $a^{2}$ field and the backward-time $d^{2}$ field (see Section 7 for notation and definitions). The $a^{2}$ field, shown in Fig. 15, is to be compared with the forward-time hyperbolicity time picture in Fig. 11, while the $d^{2}$ field of Fig. 16 is to be compared with the backward-time coherent structure boundary image in Fig. 13. When

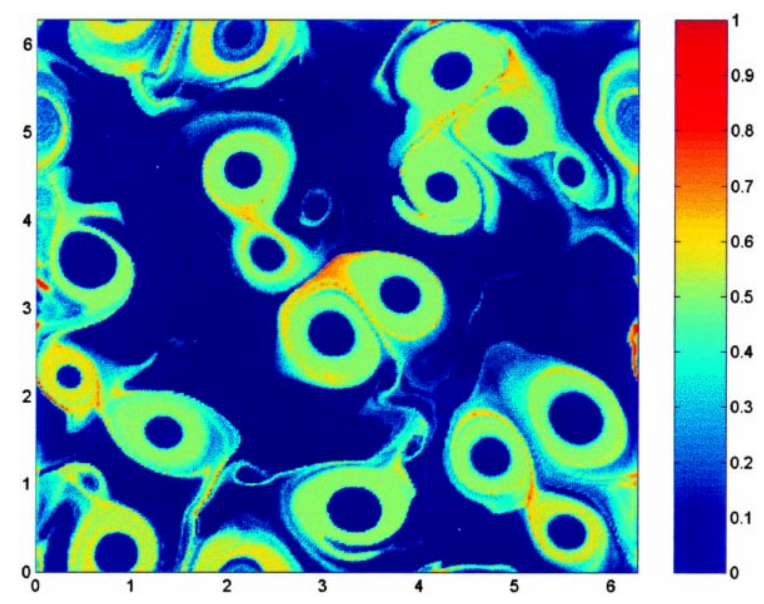

Fig. 14. The distribution of the instability time field $T_{\mathrm{u}}\left(\mathbf{x}_{0}, 50\right)$ for the hyperbolicity threshold $\lambda_{\min }=1.5$.

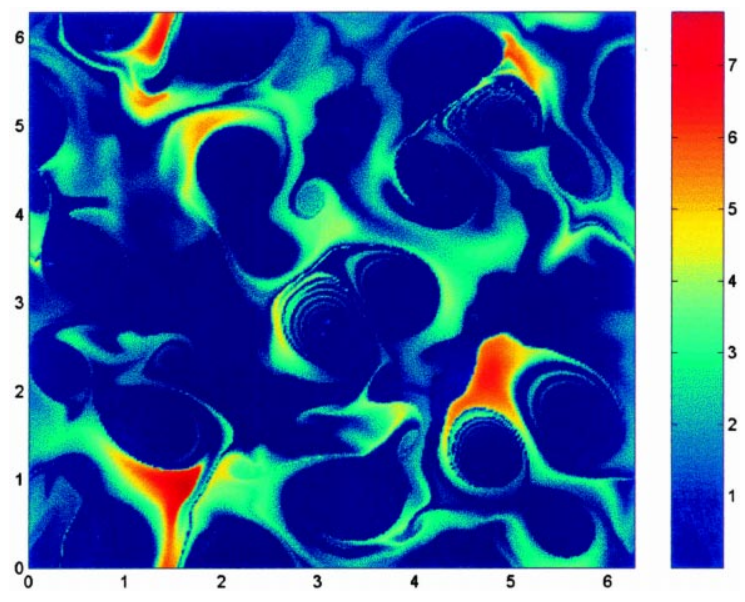

Fig. 15. The absolute dispersion density field $a^{2}\left(t, \mathbf{x}_{0}\right)$ at $t=99$.

compared, Figs. 15 and 16 show a definite correlation between dynamically and statistically defined Lagrangian coherent structure boundaries, in agreement with our discussion in Section 7. However, in contrast with the somewhat fuzzy curves obtained from relative dispersion statistics, our finite-time stability analysis yields sharp vortex boundaries whose dynamical properties are exactly known. As seen in Fig. 13, it also locates many important material lines that remain hidden to dispersion statistics calculation due to their milder rate of attraction. It is often these structures that have global connections with other regions of the

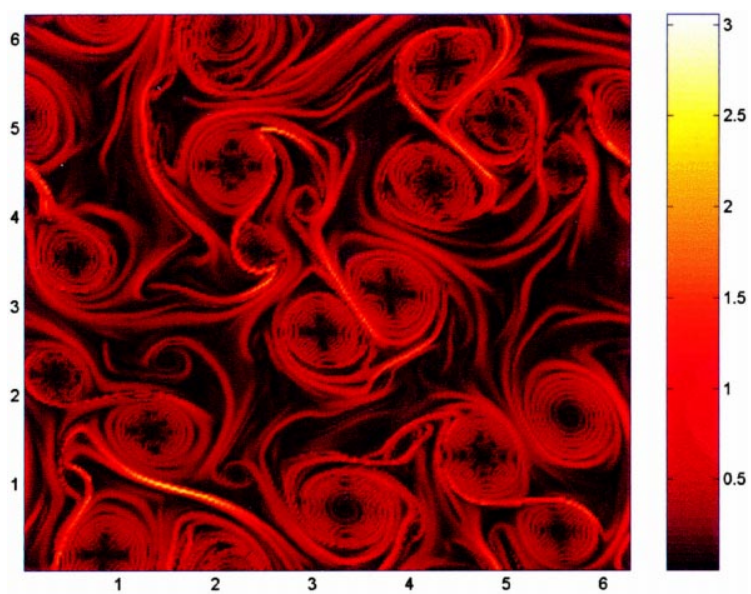

Fig. 16. The relative dispersion density field $d^{2}\left(t, \mathbf{x}_{0}\right)$ at $t=10$. (We actually plot $\sqrt{d^{2}\left(t, \mathbf{x}_{0}\right)}$ for better visibility of the structures.) 
physical space, and hence their detection is essential for understanding global mixing.

Also notable is the inability of relative dispersion to highlight vortex edges. Instead of locating the distinct closed edges obtained in Fig. 13 and Fig. 16 actually suggests the incorrect conclusion that the largest coherent structure boundaries invariably spiral towards the cores of elliptic regions. This fact would contradict the well-known impermeability of coherent vortices in two-dimensional turbulence (see, e.g., [14]), and is also inconsistent with our earlier plot of $|\nabla q|$. The spirals are, in fact, due to a shortcoming of statistical methods that we pointed out in Section 8: They are unable to distinguish between tangential and normal instabilities of material lines. As a result, they highlight shear instabilities within vortex cores as continuations of normally unstable material lines outside vortex cores.

\section{Conclusions}

In this paper we proposed a dynamical definition for coherent structure boundaries. For open flows, the boundaries are defined by material lines that are linearly stable or unstable in normal directions for longer times than their neighbors. For flows with no-slip boundary conditions, coherent structure boundaries attached to the wall (near separation or reattachment points) are defined by material lines with locally the shortest linear stability or instability times. We gave an analytic criterion that can be used to extract Lagrangian coherent structure boundaries with high precision. Our criterion can be viewed as a mathematically exact version of the Okubo-Weiss criterion for the detection of hyperbolic particle behavior. It is also Galilean invariant, and hence does not require locating instantaneous stagnation points in the flow.

To test our concepts and results, we performed numerical simulations on a velocity field arising in two-dimensional barotropic turbulence. We showed that the structure boundaries we defined coincide with those inferred from dispersion statistics. However, our computations give sharp boundaries, whose dynamical properties are exactly known by definition. In addition, our techniques reveal mesoscale structures that appear to remain completely hidden to statistical methods. The numerical algorithm we developed can also be used to isolate vortex cores and regions with different hyperbolicity levels within the background turbulence.

It appears that the framework we developed here enables one to obtain a variational principle for coherent structure boundaries. In particular, one can look for coherent structure boundaries as curves that locally maximize the hyperbolicity time distribution $T_{\mathrm{u}}\left(\mathbf{x}_{0}\right)$ defined in Section 11. A similar problem, the extraction of linear structures from visual data, arises in computer vision and is solved through an appropriate variational formulation. The available results indicate that an automated extraction of coherent structure boundaries from the hyperbolicity time distribution field is possible (see [13]). The investigation of this issue is a subject of current research.

While the primary motivation of this paper was physical, we feel that our definition of coherent structure boundaries also opens a mathematical link between the underlying partial differential equation generating the turbulent flow field and Lagrangian mixing. In particular, the quantities appearing in Theorem 1 can be translated, at least locally in time, to expressions involving the velocity field $\mathbf{u}$ and its derivatives. It is then a feasible to study the PDE governing the evolution of $\mathbf{u}$ in time, and estimate the evolution of spatial regions which contain coherent structure boundaries. A first step in this direction has been taken by Larcheveque [23], where the relationship between the Okubo-Weiss parameter $Q$ and the Navier-Stokes equation is investigated.

We finally note that our approach to defining and locating distinguished material lines can be extended to three-dimensional turbulence. In that case coherent structure boundaries will again be sought as local minimizers or maximizers of the hyperbolicity time fields. The resulting boundaries can be material lines or material surfaces, determined by a set of conditions involving the time-dependent invariants of the tensor $\nabla \mathbf{u}(\mathbf{x}(t), t)$. While the analysis is more involved, the resulting conditions are still surprisingly simple, just as in the two-dimensional case. For details, we refer the reader to [18]. In that reference an alternative ap- 
proach, based on a direct computation of Lyapunov exponents from particle paths, is also shown to produce sharp Lagrangian coherent structure boundaries.

\section{Acknowledgements}

We would like to thank Chris Jones, Leonid Kuznetsov, Jeff Weiss, and Sean Winkler for stimulating conversations, and the anonymous referees for their comments and suggestions. We also acknowledge a very helpful discussion with Norman Zabusky, who first suggested a comparison of Lagrangian coherent structure boundaries and the Okubo-Weiss parametrization of two-dimensional turbulence. We are grateful to Andrew Poje who made the velocity field used in this paper available to us. The velocity data was originally generated by the turbulence solver of Antonello Provenzale, whom we would like to thank for his help. We also thank Pat Miller for his continued help with VFTOOL and David Mumford for bringing Ref. [13] to our attention. This research was supported by ONR Grant No. N00014-93-I-0691. In addition, G. Haller was partially supported by NSF Grant No. DMS-98-00922, AFOSR Grant No. F49620-00-1-0133, and an Alfred P. Sloan Fellowship.

\section{Appendix A}

Here we give a precise definition of finite-time uniform hyperbolicity for trajectories of the velocity field (1) and sketch the proof of our main theorem in Section 9.

First, recall that by incompressibility, any linearly unstable piece of a material surface must contain exponentially converging trajectories. As a result, on the instability interval $I_{\mathrm{u}}$, an unstable material surface necessarily contains "saddle-type" trajectories. Searching for the linearly unstable portions of material surfaces is therefore equivalent to searching for trajectories that are of saddle nature on arbitrary short time intervals within $I_{\mathrm{u}}$.

In [17], the main focus was precisely the detection of such uniformly finite-time hyperbolic trajectories. Here we first recall the exact definition of uniform finite-time hyperbolicity used in [17] for general (com- pressible) flow fields of the form (1). For a fixed trajectory $\mathbf{x}(t)$, we shall use the notation $\mathbf{D}_{\tau}^{h} \equiv \nabla \mathbf{F}_{\tau}^{h}\left(\mathbf{x}_{0}\right)$.

Definition A.1. We call a trajectory $\mathbf{x}(t)$ uniformly hyperbolic on the time interval $I_{\mathrm{u}}$ if for some constants $0<\lambda, \mu<1$ and for sufficiently small $h>0$, there exist two families of one-dimensional subspaces $E_{\tau}^{+}(h)$ and $E_{\tau}^{-}(h)$ of $\mathbb{R}^{2}$, depending continuously on $\tau$ and $h$, such that $E_{\tau}^{+}(h) \oplus E_{\tau}^{-}(h)=\mathbb{R}^{2}$, and

$\mathbf{D}_{\tau}^{h} E_{\tau}^{ \pm}(h)=E_{\tau+h}^{ \pm}(h)$,

$\left\|\left.\mathbf{D}_{\tau}^{h}\right|_{E_{\tau}^{-}(h)}\right\| \leq 1-h \lambda$,

$\left\|\left.\left[\mathbf{D}_{\tau}^{h}\right]^{-1}\right|_{E_{\tau+h}^{+}(h)}\right\| \leq 1-h \mu$

for all $\tau, \tau+h \in I_{\mathrm{u}}$.

We now recall the main theorem from [17]. We shall use the notation introduced in Section 9, the only difference being that we initially do not assume incompressibility. As a result, the eigenvalues $\lambda_{1}(t)>0$ and $-\lambda_{2}(t)<0$ of the velocity gradient along $\mathbf{x}(t)$ will typically not be equal. Accordingly, the notation for the minimum of $\lambda_{j}(t)$ over a time interval $I_{\mathrm{u}}$ will be $\lambda_{j \min }$.

Theorem A.1. Suppose that for a trajectory $\mathbf{x}(t)$ of (1) and for all $t \in I_{\mathrm{u}}$,

$\operatorname{det} \nabla \mathbf{u}(\mathbf{x}(t), t)<0$,

$\sqrt{2} \beta\left[\frac{1}{\lambda_{1 \min }}+\frac{1}{\lambda_{2 \min }}\right]<\alpha$,

$\gamma+\frac{2 \beta^{2}}{\alpha^{2} \lambda_{1 \min } \lambda_{2 \min }} \lambda_{2}(t)<\lambda_{1}(t)$,

$\gamma+\frac{2 \beta^{2}}{\alpha^{2} \lambda_{1 \min } \lambda_{2 \min }} \lambda_{1}(t)<\lambda_{2}(t)$,

with $\gamma$ defined as

$$
\begin{aligned}
\frac{1}{\alpha^{3} \lambda_{1 \min } \lambda_{2 \min }} & \left\{\sqrt { 2 } \beta \left[\alpha^{2} \lambda_{1 \min } \lambda_{2 \min }\right.\right. \\
& \left.\left.+\sqrt{2} \alpha \beta\left(\lambda_{1 \min }+\lambda_{2 \min }\right)+2 \beta^{2}\right]\right\}^{-1}(\text { A. } 5)
\end{aligned}
$$

Then $\mathbf{x}(t)$ is uniformly hyperbolic on $I_{\mathrm{u}} \cdot{ }^{4}$

\footnotetext{
${ }^{4}$ In the original formulation the theorem has an additional condition. However, this extra condition follows from (A.2), a simple fact that was overlooked in [17].
} 
The proof of Theorem A.1 is based on a careful local analysis of the flow near $\mathbf{x}(t)$. In particular, in a local coordinate system fixed to $\mathbf{x}(t)$ one can seek conditions under which the "instantaneous hyperbolicity" assured by condition (A.1) for the Eulerian frame translates to actual uniform finite-time hyperbolicity in the Lagrangian frame. In this sense, the proof is a careful mathematical study of the validity of the Okubo-Weiss criterion for hyperbolic regions (cf. Section 10). It involves the smooth extension of the finite-time velocity field to an infinite-time field, the construction of stable and unstable manifolds for this extended field using Perron's method, and the derivation of conditions ensuring uniform hyperbolicity of these manifolds on the original time interval $\left[t_{0}, t_{1}\right]$. For details, the reader is referred to [17].

To prove Theorem 1, we need to argue that the conditions of Theorem A.1 are equivalent to those of Theorem 1 in the case of incompressible flows. First, note that for incompressible flows $\lambda_{1}(t)=\lambda_{2}(t) \equiv \lambda(t)$, $\lambda_{1 \text { min }}=\lambda_{2 \text { min }} \equiv \lambda_{\text {min }}$, which gives the following simpler form for conditions (A.2)-(A.4):

$$
\begin{aligned}
& \frac{2 \sqrt{2} \beta}{\lambda_{\min }}<\alpha, \\
& \gamma+\frac{2 \beta^{2}}{\alpha^{2} \lambda_{\min }} \lambda(t)<\lambda(t) .
\end{aligned}
$$

Using (A.6), condition (A.7) can be rewritten as

$\lambda(t)>\frac{\gamma \alpha^{2} \lambda_{\min }^{2}}{\alpha^{2} \lambda_{\min }^{2}-2 \beta^{2}}$.

This last inequality holds for all $t \in I_{\mathrm{u}}$ if and only if

$\lambda_{\min }>\frac{\gamma \alpha^{2} \lambda_{\min }^{2}}{\alpha^{2} \lambda_{\min }^{2}-2 \beta^{2}}$,

or, equivalently,

$\alpha^{2} \lambda_{\min }^{2}-2 \sqrt{2} \alpha \beta \lambda_{\min }-2 \beta^{2}>0$,

where we used (A.5). Since $\lambda_{\min }>0$ and $\alpha>0$ by definition, it is simple to verify that this last inequality is equivalent to

$$
\lambda_{\min }>(2+\sqrt{2}) \frac{\beta}{\alpha} .
$$

But condition (A.8) implies (A.6), thus, for incompressible flows, the conditions of Theorem A.1 simplify to (A.1) and (A.8), as claimed in Theorem 1. Finally, (13) follows directly from the estimates in [17].

\section{References}

[1] H. Aref, Integrable, chaotic, and turbulent vortex motion in two-dimensional flows, Ann. Rev. Fluid Mech. 15 (1983) 345-389.

[2] H. Aref, S.W. Jones, S. Mofina, I. Zawadzki, Vortices, kinematics, and chaos, Physica D 37 (1989) 423-440.

[3] A. Babiano, C. Basdevant, B. Legras, R. Sadourny, Vorticity and passive scalar dynamics in two-dimensional turbulence, J. Fluid Mech. 183 (1987) 379-397.

[4] A. Babiano, C. Basdevant, P. Le Roy, R. Sadourny, Relative dispersion in two-dimensional turbulence, J. Fluid Mech. 214 (1990) 535-557.

[5] A. Babiano, G. Boffetta, A. Provenzale, A. Vulpiani, Chaotic advection in point vortex models and two-dimensional turbulence, Phys. Fluids 6 (1994) 2465-2474.

[6] C. Basdevant, T. Philipovitch, On the validity of the "Weiss criterion" in two-dimensional turbulence, Physica D 73 (1994) 17-30.

[7] R. Benzi, G. Paladin, S. Patarnello, P. Santangelo, A. Vulpiani, Self-similar coherent structures in two-dimensional decaying turbulence, J. Phys. A 21 (1988) 1221-1237.

[8] K.P. Bowman, Manifold geometry and mixing in observed atmospheric flows, Preprint, 1999.

[9] M. Brachet, M. Meneguzzi, H. Politano, P. Sulem, The dynamics of freely decaying two-dimensional turbulence, J. Fluid Mech. 194 (1988) 333-349.

[10] R. Camassa, S. Wiggins, Chaotic advection in RayleighBénard convection, Phys. Rev. A 43 (1991) 774-797.

[11] J. Cohen, G. Haller, Lagrangian coherent structures in a flow around a backward facing step, in preparation.

[12] C. Coulliette, S. Wiggins, Intergyre transport in a wind-driven, quasigeostrophic double gyre: an application of lobe dynamics, Nonlinear Proc. Geophys. 7 (2000) 59-86.

[13] C. David, S.W. Zucker, Potentials, valleys, and dynamic global coverings, Int. J. Comput. Vision 5 (1990) 219-238.

[14] D. Elhmaïdi, A. Provenzale, A. Babiano, Elementary topology of two-dimensional turbulence from a Lagrangian viewpoin and single-particle dispersion, J. Fluid Mech. 257 (1993) 533-558.

[15] N. Fenichel, Persistence and smoothness for invariant manifolds for flows, Indiana Univ. Math. J. 21 (1971) 193 225.

[16] G. Haller, A.C. Poje, Finite-time transport in aperiodic flows, Physica D 119 (1998) 352-380.

[17] G. Haller, Finding finite-time invariant manifolds in two-dimensional velocity fields, Chaos 10 (2000) 99-108 .

[18] G. Haller, Distinguished material surfaces and coherent structures in 3D fluid flows, Physica D (2000), submitted for publication. 
[19] J. von Hardenberg, F. Fraedrich, F. Lunkeit, A. Provenzale, Transient chaotic mixing during a baroclinic life cycle, Chaos 10 (2000) 122-134.

[20] P.J. Holmes, J.L. Lumley, G. Berkooz, Turbulence, Coherent Structures, Dynamical Systems, and Symmetry, Cambridge University Press, Cambridge, 1996.

[21] B.L. Hua, P. Klein, An exact criterion for the stirring properties of nearly two-dimensional turbulence, Physica D 113 (1998) 98-110.

[22] M.N. Juckes, M.E. McIntyre, A high-resolution one-layer model of breaking planetary waves in the atmosphere, Nature 328 (1987) 590-596.

[23] M. Larcheveque, The Monge-Ampere equation and two-dimensional incompressible flows, C.R. Acad. Sci. Paris, Series II 311 (1990) 33-36.

[24] M.S. Lozier, L.J. Pratt, A.M. Rogerson, P.D. Miller, Exchange geometry revealed by float trajectories in the Gulf stream, J. Phys. Oceanogr. 27 (1997) 2327-2341.

[25] R.S. MacKay, J.D. Meiss, I.C. Percival, Transport in Hamiltonian systems, Physica D 13 (1984) 55-81.

[26] M.E. McIntyre, On the Antarctic ozone hole, J. Atmos. Terr. Phys. 51 (1987) 29-43.

[27] J.C. McWilliams, The emergence of isolated coherent vortices in turbulent flow, J. Fluid Mech. 146 (1984) 21-43.

[28] J.C. McWilliams, The vortices of two-dimensional turbulence, J. Fluid Mech. 219 (1990) 361-385.

[29] I. Mezić, S. Wiggins, A method for visualization of invariant sets of dynamical systems based on the ergodic partition, Chaos 9 (1999) 213-218.

[30] P.D. Miller, C.K.R.T. Jones, A.M. Rogerson, L.J. Pratt, Quantifying transport in numerically generated velocity fields, Physica D 110 (1997) 105-122.

[31] B. Noack, A. Banaszuk, I. Mezić, Control of laminar mixing in a recirculation region, Preprint, 1999

[32] K. Ohkitani, Wavenumber space dynamics of enstrophy cascade in a forced, two-dimensional turbulence, Phys. Fluids A 3 (1991) 1598-1611.

[33] A. Okubo, Horizontal dispersion of floatable trajectories in the vicinity of velocity singularities such as convergencies, Deep-Sea Res. 17 (1970) 445-454.

[34] J.M. Ottino, The Kinematics of Mixing: Stretching, Chaos, and Transport, Cambridge University Press, Cambridge, 1989.

[35] W.B. Owens, A statistical description of the mean circulation and eddy variability in the western North Atlantic using SOFAR floats, Prog. Oceanogr. 28 (1991) 257-303.
[36] F. Paparella, A. Babiano, C. Basdevant, A. Provenzale, P. Tanga, A Lagrangian study of the Antarctic polar vortex, J. Geophys. Res. 102 (1997) 6765-6773.

[37] R.T. Pierrehumbert, Large-scale horizontal mixing in planetary atmospheres, Phys. Fluids A 2 (1991) 12501260.

[38] R.T. Pierrehumbert, H. Yang, Global chaotic mixing on isentropic surfaces, J. Atmos. Sci. 50 (1993) 2462-2480.

[39] A.C. Poje, G. Haller, Geometry of cross-stream mixing in a double-gyre ocean model, J. Phys. Oceanogr. 29 (1999) 1649-1665.

[40] A.C. Poje, G. Haller, I. Mezić, The geometry and statistics of mixing in aperiodic flows, Phys. Fluids A 11 (1999) 29632968.

[41] A. Provenzale, A. Babiano, A. Zanella, Dynamics of Lagrangian tracers in barotropic turbulence, in: $\mathrm{H}$. Chate, J.M. Chomaz, E. Villermaux (Eds.), Mixing: Chaos and Turbulence, Plenum Press, New York, 1999.

[42] A. Provenzale, Transport by coherent barotropic vortices, Ann. Rev. Fluid Mech. 31 (1999) 55-93.

[43] A.M. Rogerson, P.D. Miller, L.J. Pratt, C.K.R.T. Jones, Lagrangian motion and fluid exchange in a barotropic meandering jet, J. Phys. Oceanogr. 29 (1999) 2635-2655.

[44] V. Rom-Kedar, A. Leonard, S. Wiggins, An analytical study of transport, mixing, and chaos in an unsteady vortical flow, J. Fluid Mech. 214 (1990) 347-358.

[45] V. Rom-Kedar, Homoclinic tangles - classification and applications, Nonlinearity 7 (1994) 441-473.

[46] R.M. Samelson, Fluid exchange across a meandering jet, J. Phys. Oceanogr. 22 (1992) 431-440.

[47] T.H. Solomon, J.P. Gollub, Chaotic particle transport in time-dependent Rayleigh-Bénard convection, Phys. Rev. A 38 (1988) 6280-6286.

[48] J. Weiss, The dynamics of enstrophy transfer in twodimensional hydrodynamics, La Jolla Institute Technical Report LJI-TN-81-124, 1981.

[49] J. Weiss, The dynamics of enstrophy transfer in twodimensional hydrodynamics, Physica D 48 (1991) 273294.

[50] J.B. Weiss, E. Knobloch, Mass transport and mixing by modulated traveling waves, Phys. Rev. A 40 (1989) 25792589.

[51] S. Wiggins, Chaotic Transport in Dynamical Systems, Springer, New York, 1992. 